$62^{\text {ème }}$ Congrès de la SFCO, 03020 (2014)

DOI: $10.1051 /$ sfco/20146203020

(C) Owned by the authors, published by EDP Sciences, 2014

\title{
Hyposialie aiguë sévère associée à une candidose révélatrice d'un diabète de type II chez un patient de 33 ans.
}

\author{
Haddad S, Jean-Christophe Fricain JC, Marteau JM, Catros S.
}

UFR odontologie Bordeaux, place Ameli Raba Leon, 33000, Bordeaux, FRANCE

Le diabète sucré est défini par une hyperglycémie à jeu supérieure à $1,26 \mathrm{~g} / \mathrm{L}$ (7mmol) (OMS 1997). Le diagnostic d'un diabète de type II, s'effectue le plus souvent chez des patients de plus de 45 ans. Les circonstances de découverte sont un dépistage systématique, l'apparition d'un syndrome cardinal (polyuropolydipsie, amaigrissement, hyperphagie) ou la survenue d'une complication (aiguë ou chronique). La xérostomie est fréquemment rapportée chez les diabétiques connus (Bajaj' s 2012). En revanche la découverte d'un diabète de type II à partir d'une hyposialie sévère n'a jamais été rapportée dans la littérature.

Un adulte de 33 ans s'est présenté en consultation pour une sécheresse buccale très invalidante présente depuis trois semaines. À l'interrogatoire on ne retrouvait pas d'antécédents médico-chirurgicaux personnels et familiaux. Le patient ne prenait aucun traitement. II ne décrivait pas de xérophtalmie. II décrivait une polydipsie (plusieurs litres par jour). La déglutition d'aliments secs était impossible. À l'examen clinique il présentait une muqueuse buccale érythémateuse avec une langue dépapillée, fissuraire. Quelques dépôts pseudo membraneux blanchâtres étaient observés sur la langue et le palais. Une perlèche des commissures labiales bilatérales était présente. Les signes cliniques ont permis de poser le diagnostic de candidose endobuccale associée à une hyposialie. Un bilan biologique étiologique a été réalisé avec notamment la recherche d'une immunodépression, d'un diabète, d'un syndrome de
Gougerot-Sjögren, d'une pathologie inflammatoire et d'un diabète. Le bilan biologique était normal en dehors d'une glycémie veineuse à jeun de $4.45 \mathrm{~g} / \mathrm{l}$ qui a confirmé le diagnostic de diabète qui pouvait être évoqué sur les signes buccaux associés à la polydipsie. Une hospitalisation en endocrinologie a été programmée pour la prise en charge du diabète et un traitement antifongique local (amphotéricine B en bain de bouche 3 fois par jour) a été prescrit au patient.

La cause la plus fréquente d'hyposialie au cabinet dentaire est la prise de médicaments à effet cholinergique (antidépresseurs, antipsychotiques, diurétiques, antihistaminiques, antispasmodiques) et sympathomimétiques (antidépresseurs, antihypertenseurs, bronchodilatateurs) (Scully 2003). Les hyposialies les plus sévères s'observent lors de la destruction des glandes salivaires principales au cours du syndrome de Gougerot Sjögren ou de l'irradiation cervicofaciale (Cho'ma 2010). Dans le cas présenté, l'hyposialie était due à la déshydratation secondaire à l'hyperglycémie. Ce cas illustre la nécessité de prescrire une glycémie à jeun dans le bilan étiologique de l'hyposialie aigue sévère.

HADDAD Stéphanie haddad.steph33@gmail.com

This is an Open Access article distributed under the terms of the Creative Commons Attribution License 4.0, which permits unrestricted use, distribution, and reproduction in any medium, provided the original work is properly cited. 